\begin{tabular}{|c|c|c|}
\hline & on Ta & on W \\
\hline$\frac{\left({ }^{87} \mathrm{Rb} /{ }^{85} \mathrm{Rb}\right) \text { from nitrate }}{\left({ }^{7} \mathrm{Rb} /{ }^{85} \mathrm{Rb}\right) \text { from sulphate }}$ & $1.004 \pm 0,005$ & $1.000 \pm 0.005$ \\
\cline { 1 - 1 } & & \\
\hline$\frac{\left({ }^{87} \mathrm{Rb} /{ }^{85} \mathrm{Rb}\right) \text { from chloride }}{\left({ }^{87} \mathrm{Rb} /{ }^{85} \mathrm{Rb}\right) \text { from sulphate }}$ & $1.019 \pm 0.007$ & $1.01 \check{0} \pm 0.005$ \\
\hline$\frac{\left({ }^{87} \mathrm{Rb} /{ }^{85} \mathrm{Rb}\right) \text { from chloride }}{\left.{ }^{87} \mathrm{Rb} /{ }^{85} \mathrm{Rb}\right) \text { from nitrate }}$ & $1.011 \pm 0.007$ & $1.014 \pm 0.008$ \\
\hline
\end{tabular}

Table 2. Difference in the measured isotopic abundance of rubidium due to various chemical compounds. Given are the ratios of ${ }^{87} \mathrm{Rb} /{ }^{85} \mathrm{Rb}$ ratios obtained from the two different chemical compounds indicated in the first column. Absolute ratios and standard deviations were calculated in a corresponding manner as in Table 1 .
Our results also show possible sources of error in relative measurements. In the first place, the initial enrichment effect may introduce an error because of its unreproducibility. Secondly, the observed variation due to different filament material and different chemical compounding shows the importance of having the samples investigated under identical condi- tions. This, however, may sometimes be difficult to ascertain in the case of isotopic abundance measurements of natural samples.

For stimulating discussions, comments and suggestions we would like to thank Drs. L. T. Aldrich, P. Eberhardt, F. G. Houtermans, E. Picciotto, H. Voshage and G. W. Wetherill.

\title{
The Separation of Light Gaseous Isotopes by Mass Diffusion Columns I
}

\author{
By W. J. DE WET * and J. Los \\ FOM-Laboratorium voor Massascheiding, Kruislaan 407, Amsterdam-O., Netherlands \\ (Z. Naturforschg. 19 a, 740-746 [1964] ; eingegangen am 31. Oktober 1963)
}

\begin{abstract}
A theoretical investigation of the factors affecting the efficiencies of mass diffusion columns, with particular emphasis on columns operated with partition membranes, for the separation of light gaseous isotopes, is extensively treated. Expressions are derived for the theoretical maximum separation factor and separative power and the optimum flow profile. The effects of partition membranes on column efficiencies are discussed and equations are derived to assess the performances of such columns. It appears that the membrane position in a column is rather critical and means to obviate unfavourable effects due to this, are suggested. Existing experimental results of Gverdtsiteli et al. ${ }^{1}$ corroborate some of the main theoretical conclusions.
\end{abstract}

The elementary isotope separation effect, acting in the radial direction in a mass diffusion column (see Fig. 1), is induced by a continuous flow of vapour from a vapour supply tube to a condenser which is concentric with the vapour supply tube. The vapour stream creates a gradient of the partial pressure of the gas with which the column is filled. In the steady state a dynamic equilibrium is eventually achieved between the flow of gas moved towards the condenser by collisions with the vapour molecules and the back diffusion of the gas, due to the gas pressure gradient. Due to the differences in the diffusion rates of isotopes, lighter isotopes will generally diffuse more rapidly against the vapour and consequently become enriched to the center of the column.

\footnotetext{
* On attachment from the South-African Atomic Energy Board, Pelindaba, Pretoria, Republic of South Africa.
}

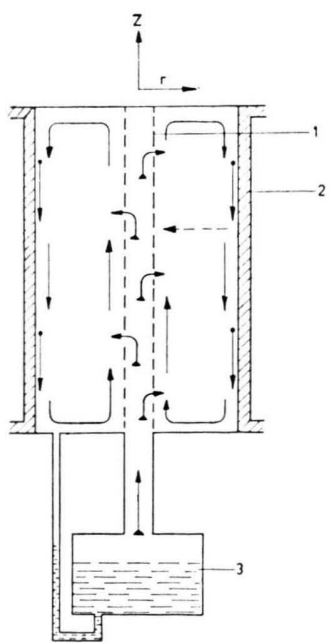

Fig. 1. Schematic diagram of a mass diffusion column. 1. porous vapour supply tube. 2 . condenser. 3 boiler. vapour stream. $\longrightarrow$ condensate. current stream. $-\longrightarrow \rightarrow$ light isotope stream. 
It is well known that if a suitable vertical countercurrent flow is established in between the vapour supply tube and the condenser, multiplication of the elementary effect takes place. In practice, however, this is rather difficult. Indeed, the various previous authors reported widely different methods for affecting such countercurrent flows. These methods involve the use of low permeability partition membranes ${ }^{1-4}$, entrainment by mechanical means ${ }^{5}$ and utilisation of the viscous dragging effect produced by a stream of liquid flowing down along the vertical condenser walls of the column like a liquid curtain ${ }^{6,7}$. A brief historic survey of mass diffusion columns and separating diffusion pumps can be found elsewhere ${ }^{8}$.

\section{Theory}

For the derivation of the partial differential equations which describe the concentration field in a mass or sweep diffusion column, some simplifying conditions are made. The mixture is supposed to be isothermal and the total pressure in the column to be constant. Furthermore it is assumed that the mixture as a whole as well as the vapour and the gas separately, can be treated as ideal gases.

According to Curtiss and Hirschfelder ${ }^{9}$, the diffusion velocities $\boldsymbol{V}_{i}$ of the three components in the ternary mixture are given by two independent relations

$\frac{n_{1} n_{2}}{D_{12}}\left(\boldsymbol{V}_{1}-\boldsymbol{V}_{2}\right)+\frac{n_{1} n_{0}}{D_{01}}\left(\boldsymbol{V}_{1}-\boldsymbol{V}_{0}\right)=-n^{2} \operatorname{grad} \frac{n_{1}}{n}$,

$\frac{n_{2} n_{1}}{D_{12}}\left(\boldsymbol{V}_{2}-\boldsymbol{V}_{1}\right)+\frac{n_{2} n_{0}}{D_{02}}\left(\boldsymbol{V}_{2}-\mathbf{V}_{0}\right)=-n^{2} \operatorname{grad} \frac{n_{2}}{n}$.

The diffusion velocities are defined with respect to the average mass velocity $\boldsymbol{v}$

$$
\boldsymbol{V}_{i}=\boldsymbol{v}_{i}-\boldsymbol{v} .
$$

The partial density of a component in grammoles per cubic centimeter is denoted by $n_{i}$; the diffusion

1 L. G. Gverdtsiteli, R. Y. Kucherov, and V. K. Tskhakaya, U.N. Peaceful Uses of Atomic Energy, II. Int. Conf. 1958.

2 G. F. Barvikh and R. Y. Kucherov, Proceedings of the AllUnion Scientific and Technical Conference on the Application of Radioactive Isotopes, Moscow 1957.

3 M. Benedict and A. Boas, Chem. Eng. Progr. 47, 51 [1951].

4 M. Benedict and A. Boas, Chem. Eng. Progr. 47, 111 [1951].

5 H. Korsching, Z. Naturforschg. 6 a, 213 [1951].

6 M. T. Cichelli, W. D. Weatherford, and J. R. Bowman, Chem. Eng. Progr. 47, 63 [1951]. coefficients $D_{i j}$ are the ordinary binary diffusion coefficients. The subscripts 0,1 and 2 refer to the vapour, the light and the heavy component of the gas respectively.

From these relations we immediately derive the elementary separation factor $\alpha_{0}$, which is the equilibrium separation factor between the two radial boundaries $r_{1}$ and $r_{2}$ of the column. In equilibrium the radial components of the molecular velocities $v_{1}$ and $v_{2}$ are zero. Combination of equations (1) and (2) for this case gives the gradient of the isotopic abundance ratio

$$
\frac{\mathrm{d}}{\mathrm{d} r} \ln \frac{n_{1}}{n_{2}}=\frac{\sigma \varepsilon}{r}
$$

where $\sigma$ is equal to $\left(u r_{2}\right) /\left(n D_{01}\right)$, in which $u$ denotes the vapour flow in moles per square centimeter at the condenser wall and $\varepsilon$ is given by

$$
\varepsilon=\left(D_{01}-D_{02}\right) / D_{02} \equiv \frac{m_{2}-m_{1}}{2 m_{1}} \frac{m_{0}}{m_{1}+m_{0}}
$$

where $m_{i}$ is the molecular weight of a component ${ }^{1,10}$.

Integration between the two boundaries gives the elementary separation factor $\alpha_{0}$

$$
\alpha_{0}=\left(r_{2} / r_{1}\right)^{\sigma \varepsilon} \text {. }
$$

In the case that the ratio $r_{2} / r_{1}$ is nearly one, the elementary separation factor reduces to

$$
\ln \alpha_{0}=\sigma \varepsilon\left(1-r_{1} / r_{2}\right) .
$$

For the subsequent development of the theory we assume that $\varepsilon \ll 1$, an assumption which is justified for isotopes except for the very lightest ones, $\mathrm{H}_{2}$ and $\mathrm{D}_{2}$. In the case when $\varepsilon$ is small the fundamental equations (1) and (2) can be transformed to two simpler expressions. The first one gives the net transport of the gas to be separated with respect to the average number velocity of the mixture, the second one gives the net transport of the light component with respect to the average number velocity of the gas. The equations are the same as those used by other investigators ${ }^{1}$

$$
\boldsymbol{\tau}-\gamma \boldsymbol{u}=-n D_{01} \operatorname{grad} \gamma,
$$

7 M. T. Cichelli, W. D. Weatherford, and J. R. Bowman, Chem. Eng. Progr. 47, 123 [1951].

8 H. London, Separation of Isotopes, George Newnes Ltd., London 1961.

9 C. F. Curtiss and J. O. Hirschfelder, J. Chem. Phys. 17, 550 [1949].

10 R. C. Reid and T. K. Sherwood, The Properties of Gases and Liquids, McGraw-Hill Book Co., Inc., New York 1958. 


$$
\begin{aligned}
n_{1} \boldsymbol{v}_{1}-N \boldsymbol{\tau} & =-n D \gamma \\
\cdot & \{\operatorname{grad} N+\varepsilon N(1-N) \operatorname{grad} \ln \gamma\} .
\end{aligned}
$$

The flow in moles per square centimeter per second of the mixture and the gas are denoted by $\boldsymbol{u}$ and $\tau$ respectively; $\gamma$ is the mole fraction of the gas in the mixture, while $N$ is the mole fraction of the light component with respect to the gas. $D$ is an effective diffusion coefficient of the light component into the mixture, given by

$$
D=\frac{D_{01}}{\left(D_{01} / D_{12}-1\right) \gamma+1} .
$$

The equations (7) and (8) can also be applied when $\varepsilon$ is not small as compared to unity, provided that $N$ is nearly one. Thus for the case of enriching deuterium in hydrogen the theory can be applied when working with very low deuterium concentrations.

For the countercurrent column we assume the radial gas velocity to be zero, neglecting the possibility of radial remixing currents. For this case equation (7) can be used to give the partial pressure of the gas as a function of the radius.

$$
\frac{\partial}{\partial r} \ln \gamma=\frac{\sigma}{r}
$$

where $u$ again is the radial flow of vapour at the condenser wall.

From equation (8) the column equation is derived taking the divergence of the flow of component one, $n_{1} \boldsymbol{v}_{1}$, which is zero for the stationary state. Bearing in mind that $\tau$ has no radial component, and putting the axial component of $\tau$ equal to $n \gamma$ times the axial component of the averaged mass velocity $w$, we get

$$
\begin{aligned}
\frac{1}{r} \frac{\partial}{\partial r} r n D \gamma\{ & \left\{\frac{\partial N}{\partial r}+N(1-N) \frac{\varepsilon \sigma}{r}\right\} \\
& +n D \gamma \frac{\partial^{2} N}{\partial z^{2}}-n \gamma w \frac{\partial N}{\partial z}=0 .
\end{aligned}
$$

This equation can be solved along the lines pointed out by Bramley et al. ${ }^{11}$, and later worked out for the case of the countercurrent gas centrifuge by CoHen ${ }^{12}$. The essential assumption of this method is that $\partial N / \partial r$ is a small quantity, which involves that $N$ and $\partial N / \partial z$ as functions of $r$ vary little as compared to $r, \gamma$ or $w$. Equation (11) can be transformed then to the ordinary column equation for the stationary state

$$
P\left(N_{\mathrm{p}}-N\right)=c_{1} N(1-N)-c_{5} \frac{\mathrm{d} N}{\mathrm{~d} z} .
$$

The column parameters for the case of mass diffusion are given by

$$
\begin{aligned}
& c_{1}=2 \pi \varepsilon \sigma \int_{r_{1}}^{r_{2}} \frac{\mathrm{d} r}{r} \int_{r_{1}}^{r} \gamma n w r \mathrm{~d} r, \\
& c_{2}=2 \pi \int_{r_{1}}^{r_{2}} \gamma n D r \mathrm{~d} r, \\
& c_{3}=2 \pi \int_{r_{2}}^{r_{1}} \frac{\mathrm{d} r}{\gamma n D r}\left(\int_{r_{1}}^{r} \gamma n w r \mathrm{~d} r\right)^{2}, \\
& P=2 \pi \int_{r_{1}}^{r_{2}} \gamma n w r \mathrm{~d} r, \\
& c_{5}=c_{2}+c_{3} .
\end{aligned}
$$

The material is withdrawn from the top of the column at a rate of $P$ moles/sec with a mole fraction $N_{\mathrm{p}}$.

The techniques for solving this equation are treated very elegantly by CoHen ${ }^{12}$. When no material is withdrawn, the separation factor $Q$ is found directly by integrating equation (12), putting $P$ equal to zero

$$
\ln Q=\frac{c_{1}}{c_{5}} l=\frac{c_{1}}{c_{2}+c_{3}} l=\frac{a_{1} L}{c_{2}+a_{3} L^{2}} l
$$

where $l$ designates the column length.

The countercurrent flow rate $L$ is defined as the total flow up and down the column.

$$
L=2 \pi \int_{r_{1}}^{r_{2}} \gamma n|w| r \mathrm{~d} r .
$$

The reduced column parameters $a_{1}$ and $a_{3}$ only depend on the flow pattern, not on the flow rate. As a function of $L$, maximum separation is obtained at $L_{0}$, for which value $c_{1} / c_{5}$ becomes $2 \varepsilon_{0}$.

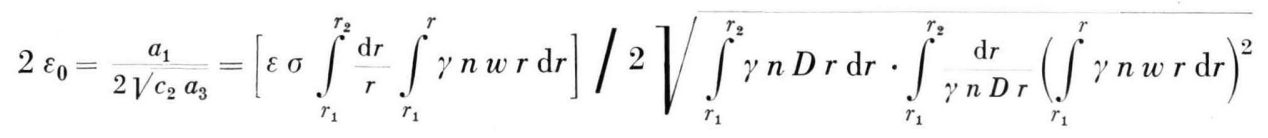

\footnotetext{
11 A. Bramley and K. Brewer, Science 92, 427 [1940].
}

12 K. Cohen, The Theory of Isotope Separation as Applied to the Large-Scale Production of $\mathrm{U}^{235}$, McGraw-Hill Book Co., Inc., New York 1951. 


$$
L_{0}=\sqrt{\frac{c_{2}}{a_{3}}}=2 \pi \int_{r_{1}}^{r_{2}} \gamma n|w| r \mathrm{~d} r \sqrt{\int_{r_{1}}^{r_{2}} \gamma n D r \mathrm{~d} r} /\left[\int_{r_{1}}^{r_{2}} \frac{\mathrm{d} r}{\gamma n D r}\left(\int_{r_{1}}^{r} \gamma n w r \mathrm{~d} r\right)^{2}\right] .
$$

Putting $L=m L_{0}$, the separation factor of equation (14) can be rewritten as

$$
\ln Q=\frac{2 m}{1+m^{2}} 2 \varepsilon_{0} l .
$$

With a production rate $P$, the solution of the column equation is given by an implicit relation between $P$ and $N_{\mathrm{p}}$.

$$
\varepsilon l \Delta(\psi)=\tanh ^{-1} \frac{\left(N_{\mathrm{p}}-N_{0}\right) \Delta(\psi)}{\left(N_{\mathrm{p}}-2 N_{\mathrm{p}} N_{0}+N_{0}\right)-\left(N_{\mathrm{p}}-N_{0}\right) \psi}
$$

where

$$
\begin{aligned}
\psi & =P / c_{1}, \\
\Delta(\psi) & =\sqrt{ } 1+2\left(1-2 N_{\mathrm{p}}\right) \psi+\psi^{2} .
\end{aligned}
$$

For this solution of the column equation several approximations are justified ${ }^{6,12}$ depending on the concentration $N$, to yield a more explicit relation between $P$ and $N_{\mathrm{p}}$. by

The separative power $\delta U$ of the column is given

$$
\delta U=\frac{c_{1}^{2}}{4 c_{5}} l=\frac{m^{2}}{1+m^{2}} \frac{c_{1}{ }^{2}}{4 c_{3}} l .
$$

The theoretical maximum separative power can be derived directly from equation (8). The separative power of an infinitesimal countercurrent element is equal to the product of the net desired material transport and the concentration gradient, divided by $N^{2}(1-N)^{2}$. Taking a thin annular slab, concentric with the axis of the column, with a radial thickness $\mathrm{d} r$ and a height $\mathrm{d} z$, as the infinitesimal element, the separative power $\mathrm{d} U$ of this element is given as

$\mathrm{d} U=$

$\frac{-\gamma n D\left\{\operatorname{grad} N+(\varepsilon \sigma / r) N(1-N) 1_{\mathrm{r}}\right\} \operatorname{grad} N}{N^{2}(1-N)^{2}} 2 \pi r \mathrm{~d} r \mathrm{~d} z$.

As a function of $\operatorname{grad} N$, the separative power is a maximum when in the radial direction

$$
\operatorname{grad} N=\frac{\varepsilon \sigma}{2 r} N(1-N) \mathbf{1}_{\mathrm{r}} .
$$

The optimum radial concentration gradient given by equation (22) is then half the equilibrium value. Substituting this value, and integrating $\mathrm{d} U$ over the volume of the column, we get the theoretical maxi- mum separative power $(\delta U)_{\max }$

$$
(\delta U)_{\max }=\frac{\varepsilon^{2} \sigma^{2} \pi l}{2} \int_{r_{1}}^{r_{2}} \frac{\gamma n D}{r} \mathrm{~d} r \mathrm{~mol} / \mathrm{sec} .
$$

The integral can be evaluated, using equations (7) and (9).

$$
(\delta U)_{\max }=\frac{\varepsilon^{2} u r_{2}}{\left(D_{01} / D_{12}-1\right)} \ln \frac{\gamma_{2}\left(D_{01} / D_{12}-1\right)+1}{\gamma_{1}\left(D_{01} / D_{12}-1\right)+1} \frac{\pi l}{2} .
$$

For high vapour consumption, and a small value of $\left(D_{01} / D_{12}-1\right)$, the expression can be approximated by

$$
(\delta U)_{\max }=\gamma_{2} \varepsilon^{2} u r_{2} \frac{1}{2} \pi l .
$$

Equation (25) reveals more clearly then the exact expression the dependance of $(\delta U)_{\max }$ on the vapour flow conditions. The vapour consumption of the column as a whole is given by

$$
U=2 \pi u r_{2} l .
$$

Substituting this in equation (25) we get the dependence of $(\delta U)_{\max }$ on the total vapour consumption

$$
(\delta U)_{\max }=\frac{1}{4} \varepsilon^{2} U .
$$

It is evident that the maximum separative power does not depend on the dimensions of the column but mainly on the total vapour consumption. Moreover, because of the linearity of separative power and total vapour consumption, the specific separative power, that is $(\delta U)_{\max } / U$, only depends on $\varepsilon$. Consequently, this method of separating isotopes by means of mass diffusion is most suitable for the lightest isotopes for which $\varepsilon$ possesses the highest values. $\varepsilon$ is also a function of the mass of the auxiliary vapour used and generally improves when using heavier molecular weight working liquids. According to equation (4) not much is apparently gained, however, by using liquids having molecular weights more than about five times the mass of the gaseous isotopes.

The optimal flow pattern can be derived from equation (11) and the condition for maximum separative power on the radial concentration gradient given in equation (22). Dropping the second order 
term in $\partial^{2} N / \partial z^{2}$, integration of equation (11) gives an expression for the radial concentration gradient.

$$
\frac{\partial N}{\partial r}=-\frac{\varepsilon \sigma}{r}+\frac{1}{r n D \gamma} \frac{\partial N}{\partial z} \int_{r_{1}}^{r} \gamma n w r \mathrm{~d} r .
$$

Following the arguments given by Los and KIsteMAKER ${ }^{13}$ for the optimal flow pattern in a countercurrent gas centrifuge, we derive for the mass diffusion column

$$
\int_{r_{1}}^{r} \gamma n w r \mathrm{~d} r \sim \gamma n D, r_{1} \leqq r<r_{2} .
$$

The downward stream has to take place as a $\delta$. function along the condenser wall, while in the upward stream $w$ is approximately $\sim 1 / r^{2}$. Using a different approach, Bock ${ }^{14}$ derived an analogous result. Substitution of the optimal velocity profile in the general expression for the separative power, equation (21), gives the right result

$$
\delta U=\frac{m^{2}}{1+m^{2}}(\delta U)_{\max } .
$$

The maximum separation factor for this optimal profile is derived from equation (16) and is given by

$$
\left(2 \varepsilon_{0} l\right)_{\max }=\frac{\varepsilon \sigma l}{2} \sqrt{\frac{\int_{r_{1}}^{r_{2}}(\gamma n D / r) \mathrm{d} r}{\int_{r_{2}}^{r_{2}} \gamma n D r \mathrm{~d} r}} .
$$

\section{Mass Diffusion Columns Operated with Partition Membranes}

As mentioned previously there are several methods of establishing the countercurrent. One method ${ }^{1-4}$, also used by us (a forthcoming paper ${ }^{15}$ covering experimental results), makes use of a thin partition membrane of low permeability in between the vapour supply tube and the condenser ${ }^{16}$. Furthermore, it fulfils no special purpose except simultaneously serving as a thin partition separating the two opposite streams.

As was pointed out by Benedict et al. ${ }^{3}$, the diffusion in a hole of the membrane can be described by the ordinary diffusion equations provided the pores are large compared to the free path length of the molecules. The membrane, however, reduces the surface area through which diffusion takes place, which is mathematically equivalent to an increase of the diffusion resistance length. The effective thickness of a membrane is thus larger than the geometrical thickness $d$, namely by a factor $1 / \lambda$, where $\lambda$ is the fraction of the surface area available to flow. Although at both sides of the membrane the velocity profile only begins to develop, we will assume, as was done by previous authors ${ }^{1,2}$, that the axial velocities are $\delta$-shaped in the two spaces. The total magnitude of the flow up and down the column is again given as $L$ moles per second and the production rate by $P$ moles per second, while $P$ is assumed to be small in comparison with $L$.

With these specifying assumptions the column parameters can be developed easily, making use of the relation between $r$ and $\gamma$ of equation (10)

$c_{1}=\frac{L \varepsilon}{2} \ln \gamma_{\mathrm{a}} / \gamma_{\mathrm{b}}$,

$c_{2}=\pi\left[\gamma_{\mathrm{a}} n D_{\mathrm{a}}\left(r_{2}{ }^{2}-r_{\mathrm{m}}^{2}\right)+\gamma_{\mathrm{b}} n D_{\mathrm{b}}\left({r_{\mathrm{m}}}^{2}-r_{1}{ }^{2}\right)\right]$, $c_{3}=\frac{L^{2}}{8 \pi u r_{2} \gamma_{\mathrm{a}}}\left[\gamma_{\mathrm{a}}\left(\frac{D_{01}}{D_{12}}-1\right) \ln \frac{\gamma_{\mathrm{a}}}{\gamma_{\mathrm{b}}}+\left(\frac{\gamma_{\mathrm{a}}}{\gamma_{\mathrm{b}}}-1\right)\right]$.

For the evaluation of $c_{2}$, which represents the axial back diffusion, integration has been done by considering average values of $\gamma n D$ at either side of the membrane, corresponding respectively to the average values of $\gamma$ at the respective sides (see Fig. 2); $r_{\mathrm{m}}$ is the radius of the membrane.

At total reflux, the separation factor of the column now follows from equations (16) and (18)

$$
\ln Q=\frac{2 m}{1+m^{2}} \frac{\ln \varepsilon \sqrt{u r_{2} \gamma_{\mathrm{a}}} \ln \left(\gamma_{\mathrm{a}} / \gamma_{\mathrm{b}}\right)}{\left[2\left\{\gamma_{\mathrm{a}} n D_{\mathrm{a}}\left(r_{2}{ }^{2}-r_{\mathrm{m}}{ }^{2}\right)+\gamma_{\mathrm{b}} n D_{\mathrm{b}}\left(r_{\mathrm{m}}{ }^{2}-r_{1}^{2}\right)\right\}\right]^{1 / 2} \times\left[\gamma_{\mathrm{a}}\left(D_{01} / D_{12}-1\right) \ln \left(\gamma_{\mathrm{a}} / \gamma_{\mathrm{b}}\right)+\left(\gamma_{\mathrm{a}} / \gamma_{\mathrm{b}}-1\right)\right]^{1 / 2}} .
$$

The separative power of the column can be found from equation (21)

$$
\delta U=\frac{m^{2}}{1+m^{2}} \varepsilon^{2} u r_{2} \frac{\gamma_{\mathrm{a}} \ln ^{2}\left(\gamma_{\mathrm{a}} / \gamma_{\mathrm{b}}\right)}{\left[\gamma_{\mathrm{a}}\left(D_{01} / D_{12}-1\right) \ln \left(\gamma_{\mathrm{a}} / \gamma_{\mathrm{b}}\right)+\left(\gamma_{\mathrm{a}} / \gamma_{\mathrm{b}}\right)-1\right)} \frac{\pi l}{2} .
$$

13 J. Los and J. Kistemaker, Proc. Symposium on Isotope Separation, North-Holland Publ. Co., Amsterdam 1958.

14 I. E. Bоск, FOM-Report, No. 13 138, 1962.
15 W. J. de Wet and J. Los, Z. Naturforschg. 19 a, 747 [1964].

${ }^{16}$ See ref. ${ }^{1}$ for an explanation and further details concerning the basic countercurrent process. 


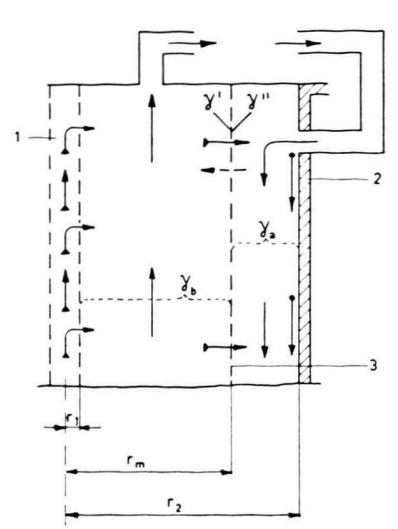

Fig. 2. Details of a mass diffusion column with a partition membrane. 1. vapour supply. 2. condenser. 3. partition membrane. $\longrightarrow$ vapour stream. $\longrightarrow$ condensate. $\longrightarrow$ countercurrent stream. $--\longrightarrow \rightarrow$ light isotope stream. $\gamma_{a}$ is the average gas mole fraction of the downward stream. $\gamma_{b}$ is the average gas mole fraction of the upward stream.

In the first place, it can be remarked that this expression for the separative power reduces to the one found by Gverdtsiteli et al. ${ }^{1}$ when $\left(D_{10} / D_{12}-1\right)$ is put equal to zero. This was presumably done by them for mathematical convenience. The influence of this term on the separation factor and separative power for small values of $\gamma_{\mathrm{a}} / \gamma_{\mathrm{b}}$ is rather important and should not be emitted. Evidence supporting its validity can also be found elsewhere ${ }^{3}$. Secondly, our approach is based on the assumption that the radial transfer of vapour and isotopes across the membrane as well as within the upward and downward stream are due to the pressure gradients described by equations (7) and (8). This means that the values of $\gamma_{\mathrm{a}}$ and $\gamma_{\mathrm{b}}$ can be obtained from equation (10) upon integration and by taking account of the resistance offered by the membrane (as is done below). Previous authors ${ }^{1,3}$, on the other hand, assigned a fixed diffusion resistance length to the membrane as well as to the two opposite streams, independent of the vapour consumption. This was originally derived from rather doubtful considerations ${ }^{3}$. Gverdtsiteli et al. ${ }^{1}$, whilst accenting such a constant value for the diffusion resistance length still applied the equivalent of equation (10) [equation (38) given in Part II $\left.^{14}\right]$, applying to plane columns, by putting $\ln \left(\gamma_{\mathrm{a}} / \gamma_{\mathrm{b}}\right)$ proportional to $u$ as follows

$$
\ln \left(\gamma_{\mathrm{a}} / \gamma_{\mathrm{b}}\right)=u S / n D_{01},
$$

where $S$ is the total diffusion resistance length. In view of the almost dynamic equilibrium conditions inferred by equations (7) and (8) to exist in columns, this does not seem justified and gives erroneous values for $\gamma_{a} / \gamma_{b}$. On the basis of their treatment a fair correlation with experimental results is only obtainable when introducing aditional mixing terms having large values. According to our approach the value assignable to diffusion resistance offered by the two opposite streams is also roughly independent of the vapour consumption. Its value is, however, a function of various column constants and need not be specified for assessing column performances. Comparatively, its value is somewhat larger than the value suggested by Benedict et al. ${ }^{3}$.

By comparing the separative power obtainable from a membrane column as given by equation (33) with the theoretical maximum separative power given by equation (25), we see that apart from the factor $m^{2} /\left(1+m^{2}\right)$, which represents the relative influence of back diffusion and tends to a limiting value for relatively large values of $m$, the factor

$$
\eta=\frac{\gamma_{\mathrm{a}} \ln ^{2}\left(\gamma_{\mathrm{a}} / \gamma_{\mathrm{b}}\right)}{\gamma_{2}\left[\gamma_{\mathrm{a}}\left(D_{01} / D_{12}-1\right) \ln \left(\gamma_{\mathrm{a}} / \gamma_{\mathrm{b}}\right)+\left(\gamma_{\mathrm{a}} / \gamma_{\mathrm{b}}-1\right)\right]}
$$

gives an additional efficiency term for this type of column. If the flow profile in the column was amenable to further description, another factor, representing the relative influence of the actual profile on the column efficiency, should have been embodied in equations (32) and (33).

It is possible to derive expressions for $\gamma_{a}$ and $\gamma_{b}$ in terms of the radial dimensions of the column and of the vapour consumption by utilising equation (10). If $\gamma^{\prime}$ and $\gamma^{\prime \prime}$ represent the mole fractions of gas on the two opposite sides of the membrane (see Fig. 2), integration yields the following equations

$$
\begin{aligned}
\ln \left(\gamma^{\prime \prime} / \gamma^{\prime}\right) & =\sigma d / r_{\mathrm{m}} \lambda, \\
\gamma^{\prime \prime} & =\gamma_{2}\left[1-\frac{\gamma_{\mathrm{a}}\left(r_{2}{ }^{2}-r_{\mathrm{m}}{ }^{2}\right)(\sigma+2)}{2 r_{2}{ }^{2} \gamma_{2}}\right]^{\frac{\sigma}{\sigma+2},} \\
\gamma_{\mathrm{a}} & =\frac{2 \gamma_{2}\left[r_{2}{ }^{\sigma+2}-r_{\mathrm{m}}{ }^{\sigma+2}\right]}{r_{2}{ }^{\sigma}(\sigma+2)\left(r_{2}{ }^{2}-r_{\mathrm{m}}{ }^{2}\right)}, \\
\gamma_{\mathrm{b}} & =\frac{2 \gamma^{\prime}\left[r_{\mathrm{m}} \sigma+2-r_{1}{ }^{\sigma+2}\right]}{r_{\mathrm{m}}{ }^{\sigma}(\sigma+2)\left(r_{\mathrm{m}}{ }^{2}-r_{1}{ }^{2}\right)}
\end{aligned}
$$

For columns with fixed partition membranes the value of $\gamma_{\mathrm{a}} / \gamma_{\mathrm{b}}$ is a function of the magnitude of the vapour consumption since the centre of mass with respect to the gas in a column shifts closer to the condenser (which is also the case for columns without membranes) when the vapour consumption is increased, as may be easily shown by applying 
equation (10). The "separative region" of a column is consequently determined by the vapour consumption rate or more precisely by the value of the ratio $\sigma / r_{2}$.

The influence of the vapour consumption on the separative power can be investigated graphically by using equations (33) to (37). A column similar to the one used by Gverdtsiteli et al. ${ }^{1}$ for separating neon isotopes with xylene as auxiliary vapour, was chosen as a calculation example. The radial dimensions, $r_{1}, r_{\mathrm{m}}$, and $r_{2}$, were $1.3,1.65$, and $2 \mathrm{~cm}$ respectively, and the value of $d / \lambda$ was $0.6 \mathrm{~cm} . \gamma_{2}$ was assumed to be approximately unity which is indeed the case. The values of $D_{01}$ and $D_{12}$ for this system are approximately 0.15 and $0.54 \mathrm{~cm}^{2} / \mathrm{sec}$, respectively, and $\varepsilon$ is equal to 0.039 . Using these data and remembering that the separative power of a column operated under symmetrical take-off conditions (viz., when product and waste streams are about equal) yields only $80 \%$ of its maximum separative power ${ }^{17}$, the calculated results could simultaneously be compared with the experimental results of these authors. The results are given in Fig. 3 .

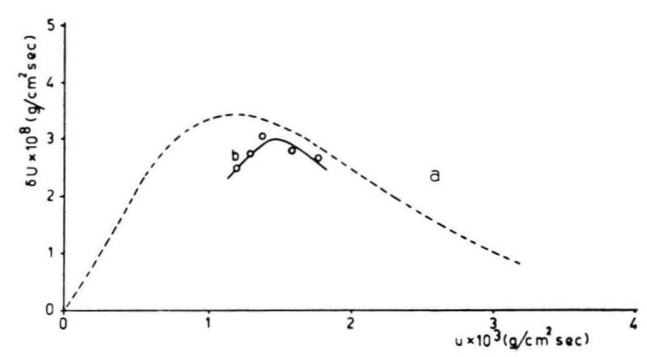

Fig. 3. Illustration of the effect of the vapour consumption on the separative power (given per unit condenser area) of mass diffusion columns with fixed membranes. a: calculated curve, b) experimental curve.

The agreement of the results in Fig. 3 is quite satisfactory if it is remembered that no corrections for adverse mixing, as inevitably always present in columns, to some extent, and no flow profile effects, were brought into account. The optimum limiting separative power is, however, attained at a somewhat higher vapour consumption rate than that predicted by the theoretical curve. This can be ascribed to the finite thickness of the film of condensed liquid running down the condenser walls which has the favourable effect of slightly reducing the width of the downward stream when the vapour consumption is increased.

The overall effect of increasing the vapour consumption is thus initially to increase the separative power almost directly proportionally. For still higher vapour consumption, the decreasing effect of high vapour consumptions on $\eta$ becomes so significant that an optimum value for the separative power is eventually reached. At even higher vapour consumptions $\eta$ decreases so pertinently that it completely overshadows the favourable effect expected from enhanced vapour consumptions. This will be more pronounced for larger values of $d / \lambda$. Fortunately, this hampering effect can be greatly eliminated by shifting the membrane closer to the condenser to maintain to low values of $\gamma_{\mathrm{a}} / \gamma_{\mathrm{b}}$, when necessary. In practice, however, columns with fixed membrane positions are obviously preferred. This implies that columns should be designed judiciously and operated with a fixed and preferably high vapour consumption, to enable high separative powers and efficiencies from a single column. High vapour consumptions are furthermore advantageous (or rather large values of $\sigma$ ), according to equation (10), to limit the amount of gas diffusing into the vapour supply tube to a negligible low fraction of the gas present in the column (see Part II). According to equations (24), (25) and also (33) the fraction of gas in the vapour supply tube should also be small to utilise the maximum separative power available from a column.

17 J. Los, D. Sc.-thesis, University of Leyden, 1963. 